\title{
Predictive Factors of Early Recurrence in Patients with Phyllodes Tumor of the Breast
}

\author{
D Bharadhwaj Ravindhran, (10) Sendhil Rajan \\ Clinic of General Surgery, St. John’s Medical College Hospital, Bangalore, Karnataka, India
}

\begin{abstract}
Objective: Phyllodes tumor (PT) is a rare entity accounting for $1 \%$ of breast neoplasms with a high propensity of recurrence. This study aimed to identify factors that are predictive of early recurrence in patients with PT.

Materials and Methods: This study reviewed clinical data of patients with PT ( $\mathrm{n}=57)$ treated at our tertiary care referral center in South India between February 2010 and December 2019. The Pearson $\chi^{2}$ test was used to investigate the relationship between patient's clinical features and tumor histotypes. Survival curves were obtained using the Kaplan-Meier method based on the log-rank test. Multivariate Cox regression analyses were performed to identify predictors of early recurrence or local recurrence-free-interval (LRFI).

Results: The mean age was 38.3 [standard deviation $(\mathrm{SD})=13.6$ ] years, and the mean follow-up was $18(\mathrm{SD}=13.5)$ months. The median tumor size was 5 $\mathrm{cm}$ (interquartile range 3 and range: $3-22 \mathrm{~cm})$. Moreover, $64.9 \%(\mathrm{n}=37)$ of the tumors were benign, $21.1 \%(\mathrm{n}=12)$ were borderline, and $14 \%(\mathrm{n}=8)$ were malignant. Of the 57 patients, $17(29.8 \%)$ developed local recurrence and one developed distant metastasis. Of the 17 patients, three were unwilling to undergo completion surgery. The median LRFI was 20 (range: 7-60) months. Multivariate cox regression analyses showed that mitotic rate $>10 /$ high power field [hazard ratio (HR) 0.147; $\mathrm{p}=0.04$ ], stromal overgrowth (HR: 4.904; $\mathrm{p}=0.05$ ), margin status (HR: 0.037; $\mathrm{p}<0.001$ ), and preoperative neutrophil-tolymphocyte ratio [(NLR), HR: 4.891; $\mathrm{p}=0.04)$ ] were significant predictors of LRFI.
\end{abstract}

Conclusion: A high mitotic rate, positive margin, stromal overgrowth, and NLR $>3.5$ were associated with early recurrence. These attributes mandate stringent follow-up, especially in a resource-limited setting.

Keywords: Aftercare, local neoplasm recurrence, phyllodes tumor

Cite this article as: Ravindran B, Rajan S. Predictive Factors of Early Recurrence in Patients with Phyllodes Tumor of the Breast.

Eur J Breast Health 2021; 17(1): 10-14.

\section{Introduction}

Phyllodes tumor (PT) is a rare neoplasm that accounts for only $1 \%$ of all breast neoplasms in women $(1,2)$. The World Health Organization (WHO) Classification of Tumors of the Breast distinguishes three histological subtypes of PTs: benign, borderline, and malignant (3). The biological behavior, clinical course, and recurrence rates of the three subtypes of PT vary widely among different reports (4-6). Most studies have investigated different cohorts of patients with various prognostic factors which could possibly predict the aforementioned outcomes (7-10).

Close follow-up of patients, especially those with tumors such as PT which have a high recurrence rate, is critical for optimal outcomes (11). However, in low- and middle-income countries (LMIC), follow-up of patients is poor because of many reasons. Therefore, identification of patients who are at a higher risk of early recurrence may help in decreasing morbidities associated with PT. Therefore, this study aimed to investigate possible predictive factors that may influence early recurrence or local recurrence-free interval (LRFI) in PT.

\section{Materials and Methods}

\section{Patient Selection}

This historical cohort study included all patients diagnosed with PT at our tertiary care referral center between February 2010 and December 2019 with complete clinicopathological data and follow-up records. All factors including age, tumor size, pathological parameters (e.g., stromal hypercellularity, mitosis, stromal atypia, stromal overgrowth, borders, necrosis, hemorrhage, epithelial hyperplasia, presence of giant cell tumors, and pathologic mitosis), histotype, local recurrence sites, and distant metastasis sites were recorded.

\section{Corresponding Author:}

Sendhil Rajan; sendhil1986@gmail.com 
The surgical approaches were classified into excision, wide local excision (WLE), mastectomy, and mastectomy with axillary clearance. Excision [performed for apparently benign findings, based on investigations such as fine-needle aspiration cytology (FNAC) and/ or ultrasonography] refers to enucleation or removal of the tumor, with margins of $<1 \mathrm{~cm}$; WLE means that the entire tumor was completely dissected with the intention of taking a rim of breast tissue using the no-see technique, with clear margins of at least $\geq 1$ $\mathrm{cm}$. The histopathological diagnoses of all cases were assessed based on established histological criteria defined by the WHO Classification of Tumors of the Breast in 2012 (3). The margin status was determined as follows: a positive margin was defined as the presence of tumor cells at the surgical margin, a close margin was defined as the presence of tumor cells $<1 \mathrm{~cm}$ from the closest surgical margin, and a clear margin was defined as the presence of tumor cells $>1 \mathrm{~cm}$ from the closest surgical margin. LRFI was defined as the period from the date of surgery to the date of diagnosis of local recurrence.

Approval of the institutional ethics review board of our institution was taken along with a waiver of consent due to the retrospective study design.

Based on previous studies (12-14) and their recommendation for axillary clearance in clinically detected lymph nodes in PT $(7,15)$, we decided to perform lymph node dissection in all patients with borderline/malignant disease and palpable lymph nodes.

\section{Protocol for Patients who Developed Recurrence}

Completion mastectomy was performed in patients who underwent WLE. In patients who underwent mastectomy, chest wall excision with margins $>1 \mathrm{~cm}$ was also performed. These patients also underwent reconstruction if required. Patients with aggressive tumors on histopathology were treated with radiation therapy based on the discussion with the multidisciplinary tumor board.

\section{Statistical Analysis}

Statistical analysis was performed using SPSS Statistics version 16.0 software (SPSS Inc., Chicago, IL, USA). The Pearson $\chi^{2}$ test was used to investigate the relationship between categorical variables. Survival curves were obtained using the Kaplan-Meier method based on the log-rank test. Univariate and multivariate cox regression analyses were performed to identify variables that were predictive of LRFI, and $\mathrm{p}<0.05$ was considered significant.

\section{Results}

The mean age of the cohort was 38.3 [standard deviation (SD): 13.6, range: 13-67] years, and the mean follow-up duration was 18 $(\mathrm{SD}=13.5)$ months. The median tumor size was $5 \mathrm{~cm}$ [interquartile range (IQR 3), range: 3-22 cm]. Moreover, 64.9\% ( $\mathrm{n}=37)$ of the tumors were benign, $21.1 \%(n=12)$ were borderline, and $14 \%(n=8)$ were malignant. Of the 57 patients, $42.1 \%(n=24)$ underwent WLE, $26.3 \%$ ( $n=15)$ (who had benign findings on FNAC) underwent excision, and $31.6 \%(\mathrm{n}=18)$ underwent mastectomy.

\section{Key Points}

- $\quad$ PT is associated with a high rate of recurrence, and identification of patients who are at a higher risk of developing early recurrence could help in decreasing morbidities associated with PT.

- Our historical cohort analysis of a series of large PTs show that mitotic rate $>10 /$ high power field (hpf) [hazard ratio (HR): 0.147 ; $\mathrm{p}=0.04]$, stromal overgrowth (HR: 4.904; $\mathrm{p}=0.05$ ), margin status (HR: 0.037; $\mathrm{p}<0.001$ ), and preoperative NLR (HR: 4.891; $\mathrm{p}=0.04$ ) were significant predictors of early recurrence.

- Identification of these factors and stringent follow-up could help in early identification of recurrence, especially in a resource-limited setting such as in our center where patient compliance to regular follow-up is still a problem.

Of the 57 patients, 17 (29.8\%) developed local recurrence. More than half $(9 / 17)$ of the patients who had a recurrence had FNAC findings suggestive of fibroadenoma or benign disease. One patient with local recurrence also developed distant metastasis. No significant differences were found between the groups with respect to the age at diagnosis or laterality between the groups. Of the 17 patients, three were unwilling to undergo completion surgery. In patients who developed local recurrence, the median age at diagnosis of the primary tumor was 42 (IQR 21) years, the median duration prior to presentation was 134 (IQR 309) days, and the median size of the primary tumor was 7 (range: $3-22) \mathrm{cm}$. Moreover, $41 \%(\mathrm{n}=7)$ of recurrent tumors were benign, 29.4\% ( $n=5)$ were borderline, and $29.4 \%(n=5)$ were malignant. The median LRFI was 20 (range: 7-60) months (Table 1). Multivariate Cox regression analyses showed that mitotic rate $>10 /$ hpf (HR: 0.147; p=0.04), stromal overgrowth (HR: 4.904; $\mathrm{p}=0.05$ ), margin status (HR: 0.037; $\mathrm{p}<0.001$ ), and preoperative NLR (HR: 4.891; $\mathrm{p}=0.04$ ) were significant predictors of LRFI (Table 2). Survival curves are shown in Figure 1.

\section{Discussion and Conclusion}

Previous reports have shown that the local recurrence rates of PTs ranged from $12 \%$ to $32 \%$ (average $-15 \%$ ). In this study, the recurrence rate at our center is almost twice the average $(6,8,9,16,17)$. This finding may be attributable to the larger tumor size at presentation, longer duration of lump, and aggressive tumor biology. In this study, more than one-fourth of the patients had a preoperative benign FNAC, thus influencing not only the type of surgery (excision vs WLE), but also the extent of surgical margin. In our cohort, the local recurrence rates were $18.9 \%(n=7), 41.6 \%(n=5)$, and $62.5 \%(n=5)$ for the benign, borderline, and malignant subtypes, respectively.

According to a multivariate cox regression analysis, the predictive factors for LRFI were high mitotic rate, stromal overgrowth, NLR $>3.5$, and margin status. The HR of 4.90 for stromal overgrowth was the highest among the four factors closely followed by NLR $>3.5(\mathrm{HR}=4.89)$. It appears that the stromal component significantly affects the recurrence and LRFI in PTs. The margin status, which is an indicator of adequate surgical clearance $(\mathrm{HR}=3.79)$, was also an important factor for LRFI.

To our knowledge, this study is one of the first to investigate the LRFI in PT and could possibly help in recognizing patients at a higher risk of developing early recurrence following surgery for PT. Patients with the above risk factors could be followed up closely. Patients with an aggressive tumor type with close or positive margins should undergo 


\section{Table 1. Comparison of attributes between patients with or without tumor recurrence}

\begin{tabular}{|c|c|c|c|}
\hline Feature & $\begin{array}{l}\text { Patients without } \\
\text { recurrence } \\
(n=40)\end{array}$ & $\begin{array}{l}\text { Patients with } \\
\text { recurrence } \\
(n=17)\end{array}$ & $\mathbf{p}$ \\
\hline \multicolumn{4}{|l|}{ Age at diagnosis } \\
\hline Median (IQR) & $34.5(17.5)$ & $42(21.5)$ & 0.16 \\
\hline Duration of lump, median (IQR) & $40(65.5)$ & $134(309)$ & 0.01 \\
\hline Left breast & $24(60 \%)$ & $9(52.9 \%)$ & \multirow{2}{*}{0.77} \\
\hline Right breast & $16(40 \%)$ & $7(47.1 \%)$ & \\
\hline Tumor size-Largest dimension $(\mathrm{cm})$, mean \pm SD & $5.15 \pm 2.12$ & $8.6 \pm 5.5$ & \multirow[t]{2}{*}{0.008} \\
\hline Benign & $30(75 \%)$ & $7(41.2 \%)$ & \\
\hline Borderline & $7(17.5 \%)$ & $5(29.4 \%)$ & \multirow[t]{2}{*}{0.03} \\
\hline Malignant & $3(7.5 \%)$ & $5(29.4 \%)$ & \\
\hline Lumpectomy & $14(35 \%)$ & $1(5.9 \%)$ & \multirow{4}{*}{0.005} \\
\hline Wide local excision & $18(45 \%)$ & $6(35.2 \%)$ & \\
\hline Mastectomy & $4(10 \%)$ & $7(41.2 \%)$ & \\
\hline Mastectomy with axillary clearance & $4(10 \%)$ & $3(17.6 \%)$ & \\
\hline Clear margin & $29(72.5 \%)$ & $2(11.8 \%)$ & \multirow{3}{*}{$<0.001$} \\
\hline Close margin & $8(20 \%)$ & $5(29.4 \%)$ & \\
\hline Positive margin & $3(7.5 \%)$ & $10(58.8 \%)$ & \\
\hline$N L R>3.5$ & $27(67.5 \%)$ & $6(37.3 \%)$ & \multirow{2}{*}{0.04} \\
\hline$N L R<3.5$ & $13(32.5 \%)$ & $11(64.7 \%)$ & \\
\hline \multicolumn{4}{|l|}{ Stromal overgrowth } \\
\hline Minimal & $7(17.5 \%)$ & $2(11.8 \%)$ & \multirow{3}{*}{0.5} \\
\hline Moderate & $24(60 \%)$ & 9 (52.9) & \\
\hline Marked & $9(22.5 \%)$ & $6(35.3 \%)$ & \\
\hline \multicolumn{4}{|l|}{ Mitotic figures/Hpf } \\
\hline $0-4$ & $29(72.5 \%)$ & $5(29.4 \%)$ & \multirow{3}{*}{0.01} \\
\hline $5-9$ & $6(15 \%)$ & $7(41.2 \%)$ & \\
\hline$>10$ & $5(12.5 \%)$ & $5(29.4 \%)$ & \\
\hline
\end{tabular}

completion surgery at the earliest, which could reduce the morbidity associated with PT.

Study limitations include a retrospective study design and associated inherent bias. Many of our patients were diagnosed to have fibroadenomas on FNAC, which was later proven to be PT. This could be the reason for a high recurrence rate in patients with a benign PT in our series. A preoperative diagnosis of fibroadenoma or a missed diagnosis of PT probably resulted in an inadequate surgical margin. Prospective validation of these data with a core-biopsy proven diagnosis of PT is necessary to confirm the efficacy of these parameters as predictors of early recurrence.

In summary, we found that stromal overgrowth, high mitotic rate, NLR >3.5 and margin status are associated with a shorter LRFI and therefore may predict earlier recurrence. The identification of these risk factors in patients with PT followed by close follow-up are critical for early recognition of local recurrence which may help improve the overall outcome, especially in an LMIC setting. 

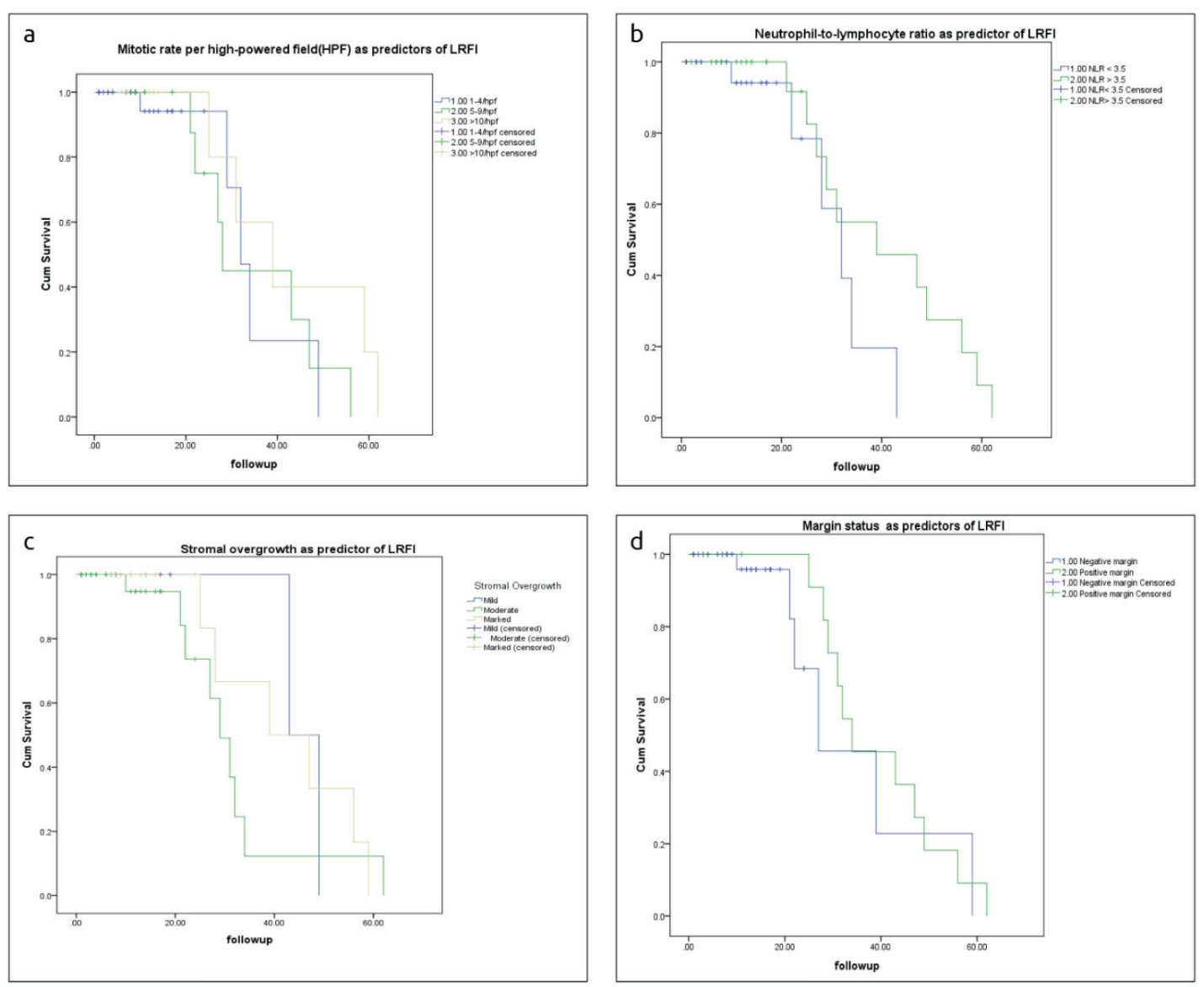

Figure 1. a-d. Local recurrence-free interval survival curves stratified by margin status, high mitotic rate, neutrophil-to-lymphocyte ratio >3.5, and stromal over growth

LRFI: Local recurrence-free interval; HPF: High-powered field; NLF: Neutrophil-to-lymphocyte; Cum: Cummulative

Table 2. Cox regression analysis of variables as predictors of local recurrence-free interval

$95.0 \% \mathrm{Cl}$ for $\operatorname{Exp}(\mathrm{B})$

Factor

Mitotic figures per high power field

Stromal overgrowth

Nuclear atypia

Cellular atypia

Close/positive margin

Neutrophil-to-lymphocyte ratio $>3.5$

Platelet-to-lymphocyte ratio $>190$

Cl: Confidence interval; Sig: Significance
Ethics Committee Approval: The study was approved by the institutional Ethics Committee of St. John's National academy of Health Sciences - (IEC letter no: 12/2018).

Informed Consent: Due to the retrospective design of the study, informed consent was not taken.

Peer-review: Externally peer-reviewed.

\section{Authorship Contributions}

Surgical and Medical Practices: B.R., S.R.; Concept: B.R., S.R.; Design: B.R., S.R.; Data Collection or Processing: B.R., S.R.; Analysis or Interpretation: B.R., S.R.; Literature Search: B.R., S.R.; Writing: B.R., S.R.

Conflict of Interest: No conflict of interest was declared by the authors.

Financial Disclosure: None for research, Travel grant awarded by BSI (Breast Surgery International) for presentation at WCS 2019, Krakow.

\section{References}

1. Belkacémi Y, Bousquet G, Marsiglia H, Ray-Coquard I, Magné N, Malard Y, et al. Phyllodes tumor of the breast. Int J Radiat Oncol 2018; 70: 492-500. (PMID: 17931796) [Crossref]

2. Suzuki-Uematsu S, Shiraishi K, Ito T, Adachi N, Inage Y, Taeda Y, et al. Malignant phyllodes tumor composed almost exclusively of a fibrosarcomatous component: a case report and review of malignant 
phyllodes tumors with metastases. Breast Cancer 2010; 17: 218-224. (PMID: 19350353) [Crossref]

3. Hanby AM, Walker C. Tavassoli FA, Devilee P. Pathology and Genetics: Tumours of the Breast and Female Genital Organs. WHO Classification of Tumours series - volume IV. Lyon, France: IARC Press; 2003: 250. [Crossref]

4. Karim RZ, Gerega SK, Yang YH, Spillane A, Carmalt H, Scolyer RA, et al. Phyllodes tumours of the breast: A clinicopathological analysis of 65 cases from a single institution. Breast 2009; 18: 165-170. (PMID: 19329316) [Crossref]

5. Taira N, Takabatake D, Aogi K, Ohsumi S, Takashima S, Nishimura R, et al. Phyllodes tumor of the breast: stromal overgrowth and histological classification are useful prognosis-predictive factors for local recurrence in patients with a positive surgical margin. Jpn J Clin Oncol 2007; 37: 730736. (PMID: 17932112) [Crossref]

6. Tan PH, Jayabaskar T, Chuah KL, Lee HY, Tan Y, Hilmy M, et al. Phyllodes tumors of the breast: The role of pathologic parameters. Am J Clin Pathol 2005; 123: 529-540. (PMID: 15743740) [Crossref]

7. Reinfuss M, Mituś J, Duda K, Stelmach A, Ryś J, Smolak K. The treatment and prognosis of patients with phyllodes tumor of the breast: an analysis of 170 cases. Cancer 1996; 77: 910-916. (PMID: 8608483) [Crossref]

8. Asoglu O, Ugurlu MM, Blanchard K, Grant CS, Reynolds C, Cha SS, et al. Risk factors for recurrence and death after primary surgical treatment of malignant phyllodes tumors. Ann Surg Oncol 2004; 11: 1011-1017. (PMID: 15525831) [Crossref]

9. Tan PH. 2005 Galloway Memorial Lecture: Breast phyllodes tumours-morphology and beyond. Ann Acad Med Singapore 2005; 34: 671-677. (PMID: 16453039) [Crossref]
10. Barrio AV, Clark BD, Goldberg JI, Hoque LW, Bernik SF, Flynn LW, et al. Clinicopathologic features and long-term outcomes of 293 phyllodes tumors of the breast. Ann Surg Oncol 2007; 14: 2961-2970. (PMID: 17562113) [Crossref]

11. Abdalla HM, Sakr MA. Predictive factors of local recurrence and survival following primary surgical treatment of phyllodes tumors of the breast. J Egypt Natl Canc Inst 2006; 18: 125-133. (PMID: 17496937) [Crossref]

12. Isik A, Grassi A, Soran A. Positive axilla in breast cancer; Clinical practice in 2018. Eur J Breast Heal 2018; 14: 134-135. (PMID: 30123877) [Crossref]

13. Norris HJ, Taylor HB. Relationship of histologic features to behavior of cystosarcoma phyllodes. Analysis of ninety-four cases. Cancer 1967; 20: 2090-2099. (PMID: 4294565) [Crossref]

14. Reinfuss M, Mituś J, Duda K, Stelmach A, Ryś J, Smolak K. The treatment and prognosis of patients with phyllodes tumor of the breast: An analysis of 170 cases. Cancer 1996; 77: 910-916. (PMID: 8608483) [Crossref]

15. Ward RM, Evans HL. Cystosarcoma phyllodes. A clinicopathologic study of 26 Cases. Cancer 1986; 58: 2282-2289. (PMID: 3019505) [Crossref]

16. Ben Hassouna J, Damak T, Gamoudi A, Chargui R, Khomsi F, Mahjoub S, et al. Phyllodes tumors of the breast: a case series of 106 patients. Am J Surg 2006; 192: 141-147. (PMID: 16860620) [Crossref]

17. Parker SJ, Harries SA. Phyllodes tumours. Postgrad Med J 2001; 77: 428435. (PMID: 11423590) [Crossref] 